\title{
Crystallographic and electrical study of the chromium substituted ferrous zinc copper ferrites
}

\author{
V M NANOTI and D K KULKARNI \\ Department of Physics, Institute of Science, Civil Lines, Nagpur 440001, India \\ Abstract. The structural and electrical properties of the oxidic spinel $\mathrm{Zn}_{0.5} \mathrm{Cu}_{0.5} \mathrm{Fe}_{x} \mathrm{Cr}_{2-x} \mathrm{O}_{4}$ \\ $(x=0.8,0.9,1 \cdot 0,1 \cdot 1)$ have been investigated through $\mathrm{X}$-ray diffraction, electrical resistivity, \\ and thermoelectric measurements. \\ $\mathrm{X}$-ray diffraction data showed formation of single spinel phase with cubic structure. D.C. \\ resistivity measurements from room temperature to $850 \mathrm{~K}$ was carried out and activation \\ energy for all the compositions evaluated. Thermoelectric measurements showed $p$-type \\ semiconducting nature in all the samples.
}

Keywords. X-ray diffraction; activation energy; Seebeck coefficient; mixed ferrites.

\section{Introduction}

Many mixed metal oxides with spinel structure were investigated as they exhibit interesting structural, magnetic and electrical properties. The spinel structure consists of a cubic close packed cage of ions with metallic ions occupying 8 tetrahedral (A) and 16 octahedral (B) interstitial sites. Properties of these spinels were controlled by the type of ions and their preferences for tetrahedral (A) and octahedral (B) sites. The $\mathrm{Zn}-\mathrm{Cu}$ ferrites have aroused considerable interest due to their interesting electrical and magnetic properties. According to Mitra et al (1992), a number of $\mathrm{Fe}^{+2}$ ions are generally formed during sintering process responsible for hopping mechanism. Van Uitert (1955) reported evaporation of $\mathrm{Zn}^{+2}$ ions from $\mathrm{Zn}$ ferrites, which increases the probability of formation of $\mathrm{Fe}^{+2}$ ions. In the present work $\mathrm{Zn}_{0.5} \mathrm{Cu}_{0.5} \mathrm{Fe}_{x} \mathrm{Cr}_{2-x} \mathrm{O}_{4}$ system was applied for the study of structural as well as electrical properties.

\section{Experimental}

Four samples of the spinel series $\mathrm{Zn}_{0.5} \mathrm{Cu}_{0.5} \mathrm{Fe}_{x} \mathrm{Cr}_{2-x} \mathrm{O}_{4}$ with $x=0.8$ to 1.1 in the steps of 0.1 were prepared by conventional ceramic technique using AR grade oxides viz. $\mathrm{Fe}_{2} \mathrm{O}_{3}, \mathrm{Cr}_{2} \mathrm{O}_{3}, \mathrm{ZnO}$ and $\mathrm{CuO}$. The oxides were mixed in proper molar proportions to yield the desired stoichiometry. The mixture was ground in acetone and presintered at $1000^{\circ} \mathrm{C}$ for $16 \mathrm{~h}$ and slowly cooled. Then the samples were again mixed properly and refired at $1000^{\circ} \mathrm{C}$ for another $100 \mathrm{~h}$ and annealed initially at the rate of $100^{\circ} \mathrm{C} / \mathrm{h}$ up to $500^{\circ} \mathrm{C}$ and then cooled naturally. The X-ray diffractograms of the powdered samples were then taken using Philips $\mathrm{X}$-ray diffractometer and $\mathrm{Cu}-\mathrm{K}$ radiation with wavelength $1.542 \mathrm{~A}$. The $\mathrm{X}$-ray patterns showed a single spinel phase with cubic structure. X-ray intensity calculations were carried out for the site distribution.

DC resistivity of the samples was measured using LCR bridges [Meghommeter Model RM 160/3 (BPL India) and Digital LCR meter VLCR-17(Vasavi Electronics)]. Pellets were prepared by mixing $4 \%$ polyvinyl acetate (PVA) as a binder under 2 
tonnes/cm pressure. Initially pellets were heated at $300^{\circ} \mathrm{C}$ for $3 \mathrm{~h}$ and then at $1000^{\circ} \mathrm{C}$ by gradual rise in temperature. The final sintering was carried out for $3 \mathrm{~h}$ and the sample was furnace cooled. A thin layer of silver paste was applied over crack free pellet for good electrical contacts. Measurements were carried out from room temperature to $850 \mathrm{~K}$. Thermoelectric measurements were carried out after sandwiching thick pellets between two copper rods from room temperature to $493 \mathrm{~K}$. $\log \rho$ vs $10^{3} / T$ and $\alpha(\mathrm{mV})$ vs $T(\mathrm{~K})$ were also plotted to find out $\Delta E$ and Seebeck coefficients respectively.

\section{Results and discussion}

$\mathrm{X}$-ray patterns of all the compounds showed a single spinel phase with cubic structure. $X$-ray intensity calculations were carried out. In such mixed systems details of oxidation states, degree of inversion, cation distribution and anionic parameter decide the intensities of reflections. The most sensitive planes were taken to be indicative of adjustment parameters for bigger size anions which were of fixed oxidation states.

As none of the compounds had high conductivity or low activation energies, possibility of mixed valences of same metal ion was remote. Intensity calculations

Table 1. Lattice parameter (a), X-ray density $(\mathrm{d} x), d_{\mathrm{B}-\mathrm{B}}$ distances, activation energy $(\Delta E)$ and Seebeck coefficient $(\alpha)$ for the system $\mathrm{Zn}_{0.5} \mathrm{Cu}_{0.5} \mathrm{Fe}_{x} \mathrm{Cr}_{2-x} \mathrm{O}_{4}$.

\begin{tabular}{lccccc}
\hline$x$ & $a(\mathrm{~A})$ & $\mathrm{d} x(\mathrm{~g} / \mathrm{cc})$ & $d_{\mathrm{B}-\mathrm{B}}(\mathrm{A})$ & $\Delta E(\mathrm{eV})$ & $\alpha(\mu \mathrm{V} / \mathrm{K})$ \\
\hline 0.8 & 8.3443 & 5.4086 & 2.9502 & 0.67 & +410 \\
0.9 & 8.3490 & 5.3792 & 2.9518 & 0.61 & +355 \\
1.0 & 8.3522 & 5.3890 & 2.9529 & 0.58 & +310 \\
1.1 & 8.3549 & 5.3929 & 2.9539 & 0.53 & +280 \\
\hline
\end{tabular}

Table 2. Comparative study of $\mathrm{X}$-ray intensities for the compound $\mathrm{Zn}_{0.5} \mathrm{Cu}_{0.5} \mathrm{Fe}_{0.9} \mathrm{Cr}_{1.1} \mathrm{O}_{4}(a=8 \cdot 3490 \pm 0.0001)$.

\begin{tabular}{|c|c|c|c|c|}
\hline $\begin{array}{c}\text { Angle } \\
2 \theta\end{array}$ & $\begin{array}{c}\text { 'd' } \\
\text { (obs) }\end{array}$ & $h k l$ & $\begin{array}{l}I / I_{\max } \\
\text { (obs) }\end{array}$ & $\begin{array}{c}I / I_{\max } \\
\text { (cal) }\end{array}$ \\
\hline $23 \cdot 1700$ & 4.8203 & 111 & 5.65 & 5.63 \\
\hline $38 \cdot 2700$ & 2.9531 & 220 & $50-84$ & $50 \cdot 79$ \\
\hline $45 \cdot 1725$ & 2.5204 & 311 & $100 \cdot 00$ & $100 \cdot 00$ \\
\hline $47 \cdot 3600$ & $2 \cdot 4102$ & 222 & 5.03 & 5.02 \\
\hline $55 \cdot 2225$ & 2.0886 & 400 & 10.72 & $10-70$ \\
\hline $69 \cdot 1825$ & 1.7051 & 422 & 6.08 & 6.05 \\
\hline $74 \cdot 2050$ & 1.6047 & 333 & $14 \cdot 19$ & 14.02 \\
\hline $81 \cdot 9100$ & 1.4768 & 440 & $15 \cdot 93$ & 15.91 \\
\hline $94 \cdot 2875$ & $1 \cdot 3205$ & 620 & 2.06 & $2 \cdot 06$ \\
\hline 99.0150 & 1.2729 & 533 & $3 \cdot 38$ & $3 \cdot 36$ \\
\hline $100 \cdot 6050$ & $1 \cdot 2581$ & 622 & 2.06 & $2 \cdot 04$ \\
\hline $120 \cdot 4425$ & $1 \cdot 1153$ & 642 & $2 \cdot 19$ & $2 \cdot 15$ \\
\hline $125 \cdot 8775$ & 1.0870 & 553 & 4.83 & $4 \cdot 81$ \\
\hline
\end{tabular}


were carried out for all possible cation distributions and the highest matching intensities lead to the cation distribution with following oxidation states.

$$
\mathrm{Zn}_{0 \cdot 5}^{2+} \mathrm{Fe}_{0.5}^{3+}\left[\mathrm{Fe}_{x-0 \cdot 5}^{3+} \mathrm{Cu}_{0.5}^{2+} \mathrm{Cr}_{2-x}^{3+}\right] \mathrm{O}_{4}^{2-} \text {. }
$$

Details of observed and calculated peak intensities are listed in table 2. Typical $\mathrm{X}$-ray patterns for one of the samples is also shown in figure 2. Lattice parameters ' $a$ ' for all the compositions are listed in table 1. Lattice parameter for the compound $\mathrm{Zn}_{0.5} \mathrm{Cu}_{0.5} \mathrm{FeCrO}_{4}$ is reported as 8.3522 A (Nanoti et al 1993).

All the compounds showed room temperature resistivity of the order of $10^{4} \mathrm{ohm} . \mathrm{cm}$. The relationship

$$
\rho=\rho_{0} \exp (\Delta E / k T),
$$

[Wilson's law] is obeyed indicating semiconducting nature of all the compounds. Plots of $\log \rho$ vs $10^{3} / T$ showed no break indicating stable oxidation states. Activation
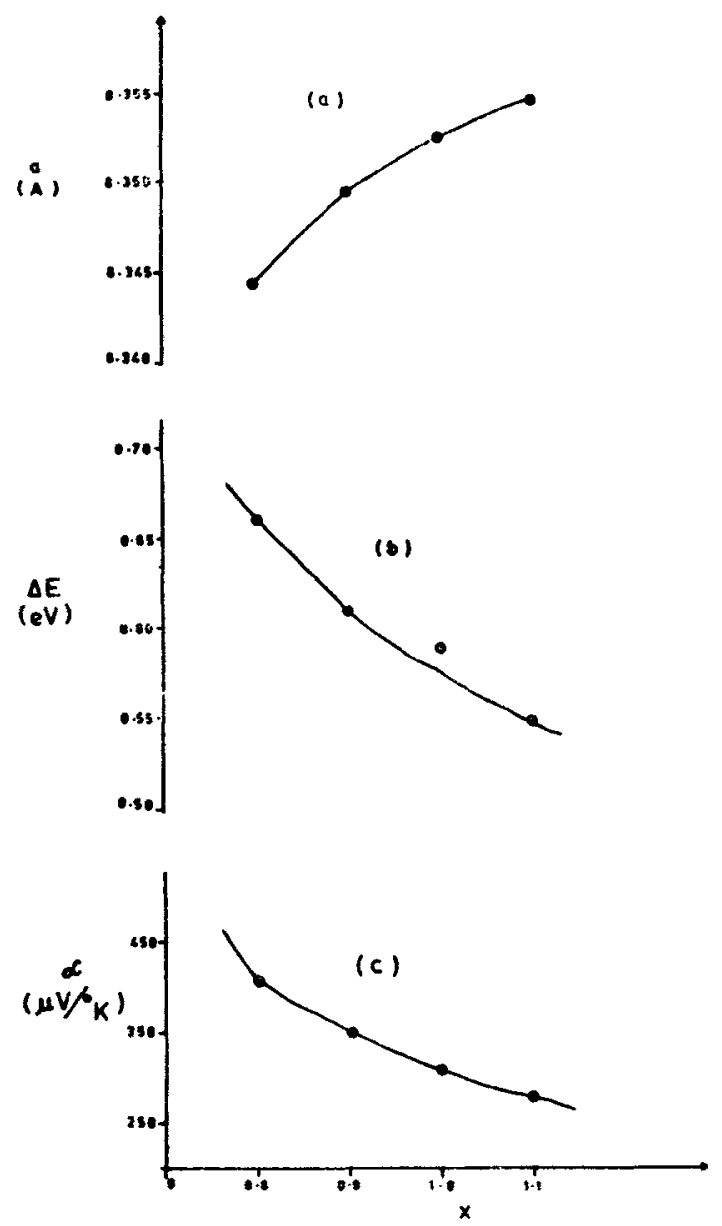

Figure 1. Plot of (a) lattice parameter ' $a$ ' vs $x$, (b) activation energy $\Delta E$ vs $x$ and (c) Seebeck coefficient $\alpha$ vs $x$. 

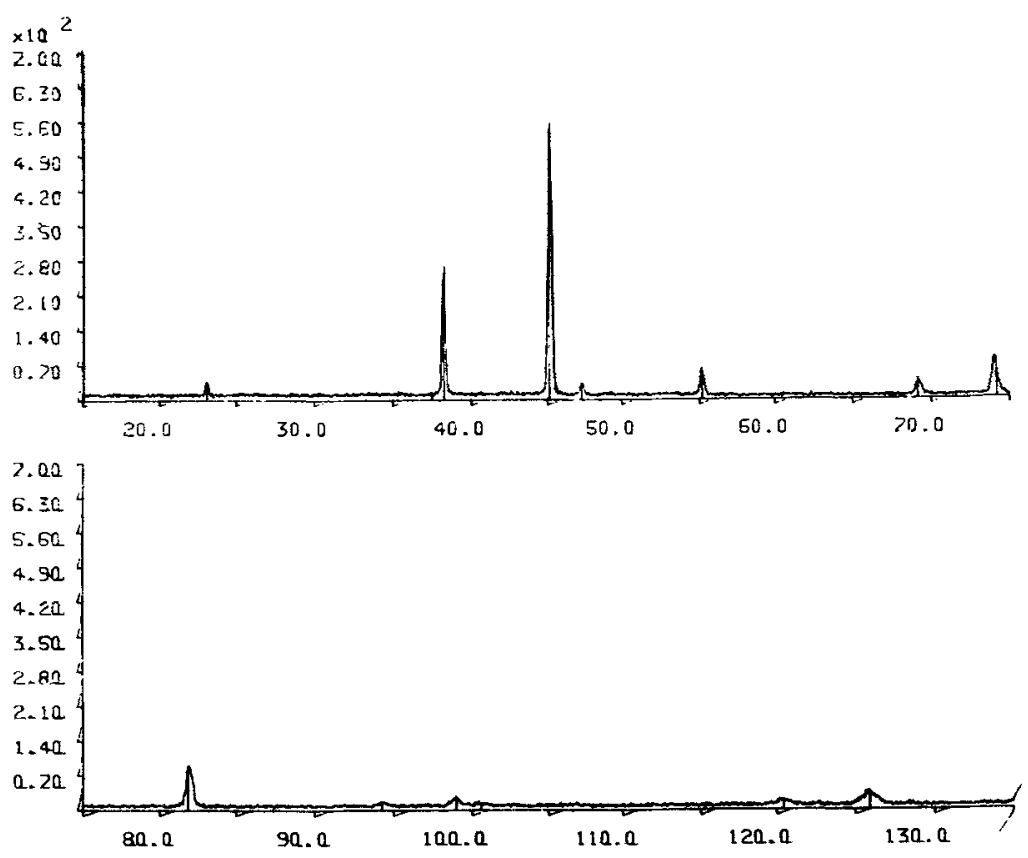

Figure 2. XRD pattern for the compound $\mathrm{Zn}_{0.5} \mathrm{Cu}_{0.5} \mathrm{Fe}_{0.9} \mathrm{Cr}_{1.1} \mathrm{O}_{4}$.

energy for the system was found to vary between $0.53 \mathrm{eV}$ to $0.67 \mathrm{eV}$ (figure 1). In spinels $d_{\mathrm{A}-\mathrm{A}}$ and $d_{\mathrm{A}-\mathrm{B}}$ distances are longer than $d_{\mathrm{B}-\mathrm{B}}$ distances. Therefore electrical conduction took place by hopping of charge carriers (holes) mostly between B-sites.

A cursory glance at table 1 shows that $E$ decreases with increasing $x$ which may be due to several reasons, such as, small number of $\mathrm{Fe}^{2+}$ ions are generally formed during sintering process, which increases the conductivity due to increased hopping between $\mathrm{Fe}^{+2}$ and $\mathrm{Fe}^{+3}$ ions (Mitra et al 1992).

Smith and Wijn (1959) and Van Uitert (1955) found that a small amount of zinc evaporates from the surface grains of zinc ferrites which increases the probability of formation of $\mathrm{Fe}^{+2}$ ions. Electrical properties of ferrites are explained on the basis of tunneling of electrons amongst $\mathrm{Fe}^{+2}$ and $\mathrm{Fe}^{3+}$ aligns on $\mathrm{B}$-sites (Srinivasan and Srivastava 1981). It has been assumed that electrons which participate in $\mathrm{Fe}^{+2} \leftrightarrow \mathrm{Fe}^{+3}+e^{-}$exchange process are strongly coupled to the lattice and tunnel from one site to other due to phonon-induced transfer mechanism, developed by Srivastava (1981). Bates and Steggels (1975) found that theoretically calculated values of resistivity and Seebeck coefficients for $\mathrm{Zn}-\mathrm{Cu}$ ferrites from the above mechanism agree well with the experimental results. Electrical conductivity and Seebeck coefficient of polycrystalline ferrous zinc ferrite and ferrous zinc copper ferrite studied as a function of temperature data have been satisfactorily analyzed on the basis of a mechanism of electron transport based on a phonon assisted electron tunneling. Conduction in the present system can be attributed to above reason. Thermo emf measurements are carried out from room temperature to $493 \mathrm{~K}$. Seebeck coefficient of the system was varied between $+280 \mu \mathrm{V} / \mathrm{K}$ to $+410 \mu \mathrm{V} / \mathrm{K}$. All compounds are 
p-type semiconductors. $p$-type semiconductivity exhibited by compounds can be attributed to loss of some $\mathrm{ZnO}$ during firing process creating vacancies at $\mathrm{A}$ site. In such a case very small amount of $\mathrm{Cu}^{+2}$ may go to A-site. Such a possibility for gallium migrating from $B$ to the $A$ site has been reported by Lotgering (1966) and O'keeffe (1961). As a result of this $\mathrm{Zn}^{+2}$ deficient compounds may be formed having vacancies at B-sites, which will trap holes giving rise to $p$-type of conduction.

\section{References}

Bates C A and Steggels P 1975 J. Phys. C8 2283

Lotgering F K 1966 Phys. Chem. Solids 27139

Mitra R, Puri R K and Mendiratta R G 1992 J. Mater. Sci. 271275

Nanoti V M, Prakash C S and Kulkarni D K 1993 Proceedings of DAE SSP symp., Bombay p. 168

O’keeffe M 1961 Phys. Chem. Solids 21172

Smith J and Wijn H P J 1959 Ferrites (New York: Wiley-Inter Science) p. 299

Srinivasan G and Srivastava C M 1981 Phys. Status Solidi b103 665

Van Uitert L G 1955 J. Phys. 31883 